\title{
Selenium-Doped Carbon Quantum Dots Efficiently Ameliorate Secondary Spinal Cord Injury via Scavenging Reactive Oxygen Species
}

This article was published in the following Dove Press journal: International Journal of Nanomedicine

\author{
Wenqi Luo ${ }^{1,2}$ \\ Yiming Wang ${ }^{1,2}$ \\ Feng Lin ${ }^{1,2}$ \\ Yixuan $\mathrm{Liu}^{2,3}$ \\ Rui Gu' \\ Wanguo Liu' \\ Chunsheng Xiao (iD ${ }^{2,4}$ \\ 'Department of Orthopaedic Surgery, \\ China-Japan Union Hospital of Jilin \\ University, Changchun I30033, People's \\ Republic of China; ${ }^{2}$ Key Laboratory of \\ Polymer Ecomaterials, Changchun \\ Institute of Applied Chemistry, Chinese \\ Academy of Sciences, Changchun I30022, \\ People's Republic of China; ${ }^{3}$ School of \\ Applied Chemistry and Engineering, \\ University of Science and Technology of \\ China, Hefei, 230026, People's Republic \\ of China; ${ }^{4}$ jilin Biomedical Polymers \\ Engineering Laboratory, Changchun \\ 130022, People's Republic of China
}

Correspondence: Wanguo Liu; Rui Gu Email liuwanguo6016@jlu.edu.cn; gurui@jlu.edu.cn
Background: The excess production of reactive oxygen species (ROS) after traumatic spinal cord injury (TSCI) has been identified as a leading cause of secondary injury, which can significantly exacerbate acute damage in the injured spinal cord. Thus, scavenging of ROS has emerged as an effective route to ameliorate secondary spinal cord injury.

Purpose: Selenium-doped carbon quantum dots (Se-CQDs) with the ability to scavenge reactive oxygen species were prepared and used for efficiently ameliorating secondary injury in TSCI.

Methods: Water-soluble Se-CQDs were easily synthesized via hydrothermal treatment of L-selenocystine. The chemical structure, size, and morphology of the Se-CQDs were characterized in detail. The biocompatibility and protective effects of the Se-CQDs against $\mathrm{H}_{2} \mathrm{O}_{2}-$ induced oxidative damage were investigated in vitro. Moreover, the behavioral test, bladder function, histological observation, Western blot were used to investigate the neuroprotective effect of Se-CQDs in a rat model of contusion TSCI.

Results: The obtained Se-CQDs exhibited good biocompatibility and remarkable protective effect against $\mathrm{H}_{2} \mathrm{O}_{2}$-induced oxidative damage in astrocytes and PC12 cells. Moreover, SeCQDs displayed marked anti-inflammatory and anti-apoptotic activities, which thereby reduced the formation of glial scars and increased the survival of neurons with unscathed myelin sheaths in vivo. As a result, Se-CQDs were capable of largely improving locomotor function of rats with TSCI.

Conclusion: This study suggests that Se-CQDs can be used as a promising therapeutic platform for ameliorating secondary injury in TSCI.

Keywords: carbon quantum dots, spinal cord injury, reactive oxygen species, antioxidant, inflammation

\section{Introduction}

Spinal cord injury can result from both traumatic and nontraumatic damage. Traumatic spinal cord injury (TSCI) occurs when an external physical impact, such as, sportsrelated injury, motor vehicle injury, fall, and violence acutely, damages the spinal cord. $^{1,2}$ TSCI usually leads to sensory, motor impairments, as well as neurogenic bladder and autonomic dysfunctions, throughout a patient's lifetime. ${ }^{3-6}$ The devastating events ultimately impact a patient's physical, psychological, and social well-being throughout the lifespan. ${ }^{7,8}$ The pathophysiological progression of TSCI consists of primary injury and secondary injury cascades. ${ }^{4,9,10}$ The primary injury occurs immediately after mechanical disruption and dislocation of the vertebral column, which 
causes compression or transection of the spinal cord. ${ }^{4,5,11}$ Immediately after the primary injury, a sustained secondary injury cascade occurs, which leads to further damage to the spinal cord and neurological dysfunction. ${ }^{4,5,10-13}$ Thus, it is of great importance to mitigate secondary injury in the clinical treatment of acute TSCI. Currently, high-dose administration of methylprednisolone sodium succinate is the only recommended neuroprotective drug available for ameliorating secondary injury after acute TSCI. ${ }^{5,14}$ However, this treatment strategy remains controversial, because while it usually leads to modest therapeutic outcomes, it is accompanied by many potential complications, such as pneumonia, infections, sepsis, pulmonary embolism, corticosteroid myopathy, and gastric bleeding. ${ }^{4,15,16}$ Therefore, exploring effective intervention methods for attenuating secondary injury associated with TSCI is urgently warranted.

Accumulating evidence suggests that the significant increase in reactive oxygen species (ROS) levels in the injured spinal cord plays a crucial role in secondary injury cascades. Excess ROS are found to be mainly generated from injured mitochondria, NADPH oxidases, inflammatory cells, and the Fenton reaction ascribed to the harsh microenvironment of secondary injury. ${ }^{5,10,17}$ The over-produced ROS can cause severe lipid peroxidation, as well as oxidative damage to proteins and DNA, resulting in the degeneration and demyelination of nerve fibers and even apoptosis of neuronal cells in the injured site. ${ }^{18-22}$ Moreover, the easy distribution of ROS into the neighboring area of the injured site often causes the expansion of the injured site, leading to aggravated secondary injury with more serious locomotion defects. $^{16,23}$ Therefore, the scavenging of ROS has been established to be an effective route to attenuate secondary injury in acute TSCI treatment. ${ }^{20,21,23-25}$

In the present study, a type of selenium-doped carbon quantum dots (Se-CQDs) was prepared and used for effectively ameliorating secondary TSCI (Figure 1). The SeCQDs were prepared via a simple approach, ie, hydrothermal treatment of L-selenocystine. ${ }^{19}$ The obtained Se-CQDs exhibited good solubility in water, good biocompatibility, and outstanding ROS scavenging ability. ${ }^{19,26}$ In addition, these Se-CQDs exhibited remarkable protection of astrocytes and PC12 cells from oxidative damage induced by $\mathrm{H}_{2} \mathrm{O}_{2}$ in vitro. Furthermore, the neuroprotective capacity of Se-CQDs in a model of contusion-induced TSCI was investigated. The results demonstrated that Se-CQDs protected the injured spinal cord by inhibiting inflammation, the demyelination of nerve fibers, and the apoptosis of neuronal cells.

\section{Materials and Methods Materials}

L-Selenocystine was purchased from Shanghai Macklin Biochemical Co., Ltd. (Shanghai, China). 3-(4,5-Dimethyl-
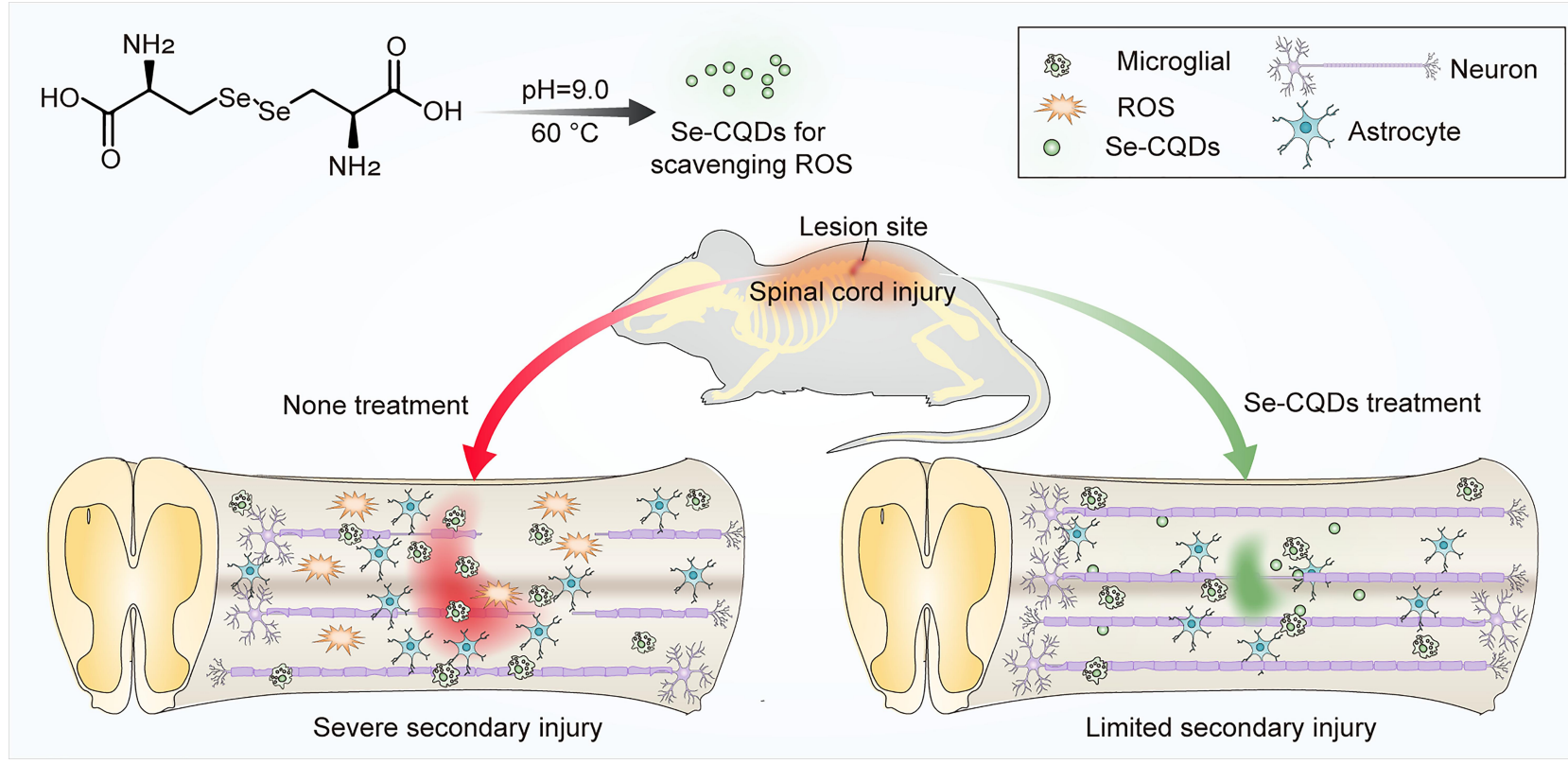

Lesion site

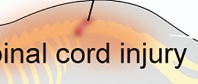

Figure I Schematic illustration of the Se-CQDs preparation for the treatment of TSCI. Water-soluble Se-CQDs were prepared by hydrothermal treatment of L-selenocystine. After intraspinal injection, the Se-CQDs efficiently scavenged ROS, consequently reducing inflammation, preventing neuronal apoptosis and demyelination, thereby improving the recovery of locomotor function of rats with $\mathrm{TSCl}$. 
thiazol-2-yl)-2,5-diphenyl tetrazolium bromide (MTT) and 4',6-diamidino-2-phenylindole dihydrochloride (DAPI) were purchased from Sigma (St. Louis, MO, USA). Dulbecco's modified Eagle's medium (DMEM) and fetal bovine serum were obtained from Gibco (Grand Island, NY, USA). Primary antibodies, including anti-glial fibrillary acidic protein (anti-GFAP), anti-neurofilament-200 kDa (anti-NF200), and anti-NeuN antibodies, were purchased from Abcam (Cambridge, UK); anti-caspase-9, anti-Bcl-2, anti-Bax, anticleaved caspase-3, and anti-GAPDH antibodies were purchased from Cell Signaling Technology (Danvers, MA, USA); and anti-chondroitin sulfate (CS56) antibodies were purchased from Sigma. Hydrogen peroxide $\left(\mathrm{H}_{2} \mathrm{O}_{2}, 30 \mathrm{wt} \%\right.$ in water) was purchased from Sinopharm Chemical Reagent Co., Ltd (Shanghai, China). A dialysis membrane was purchased from Greenbird Technology Co., Ltd (Shanghai, China). Deionized (DI) water was prepared using the MilliQ system (Millipore Co., Billerica, MA, USA). All reagents were used directly without pretreatment.

\section{Preparation and Characterization of Se-CQDs}

Water-soluble Se-CQDs were prepared by hydrothermal treatment of L-selenocystine as previously reported. ${ }^{19}$ Briefly, L-selenocystine (200 mg) was dissolved in DI water $(10 \mathrm{~mL}) . \mathrm{NaOH}$ was used to adjust the solution to $\mathrm{pH} 9$ to promote the dissolution of L-selenocystine in DI water. When the temperature of the reaction mixture reached $60^{\circ} \mathrm{C}$, the bright yellow selenocystine solution became orange-brown. The solution was then heated at $60^{\circ} \mathrm{C}$ for $24 \mathrm{~h}$. The resulting brown solution was centrifuged at 10,000 rpm, and the supernatant was collected and dialyzed $(\mathrm{MWCO}=500 \mathrm{Da})$. A brown powder was obtained after lyophilization of the Se-CQDs solution and used to further investigate its structure and physical properties. ${ }^{1} \mathrm{H}$ and ${ }^{13} \mathrm{C}$ nuclear magnetic resonance (NMR) spectra were recorded on a Bruker AV-300 NMR spectrometer (Billerica, MA, USA) in deuterated water. The particle size and size distribution of Se-CQDs were determined by dynamic light scattering (DLS) (DAWN EOS, Wyatt Technology Co., Santa Barbara, CA, USA). The zeta potential of Se-CQDs was measured on a Zetasizer Pro (Malvern Panalytical Ltd, Worcestershire, UK). The morphology of Se-CQDs was observed using a transmission electron microscope (TEM; JEOL JEM101, Tokyo, Japan). Fourier transform infrared spectra were recorded on a Bio-Rad Win-IR instrument
(Hercules, CA, USA) using the KBr method. X-ray surface photoelectron spectra were recorded on X-ray surface photoelectron spectroscopy (XPS) (Thermo ESCALAB $250, \mathrm{UK})$ after the samples were freeze-dried. The absorption spectra of Se-CQDs were measured by UV-vis Spectroscopy (Lambda 365, PerkinElmer, USA), and the fluorescence spectra of Se-CQDs were recorded on a Fluorescence spectroscopy (Hitachi, F-4600, Tokyo, Japan) in a standard quartz cuvette.

\section{Cell Culture}

Astrocytes and mouse neuroblastoma Neuro-2a (N2a) cells were obtained from the Cell Bank of the Chinese Academy of Sciences (Shanghai, China). Pheochromocytoma 12 (PC12) cells were obtained from the Basic Medical College of Jilin University. All cells were grown in DMEM (supplemented with $10 \%$ fetal bovine serum, $100 \mathrm{U} / \mathrm{mL}$ penicillin, and $100 \mathrm{U} / \mathrm{mL}$ streptomycin) at $37^{\circ} \mathrm{C}$ in an incubator with $5 \% \mathrm{CO}_{2}$ conditions and a humidified atmosphere. TrypsinEDTA was used to subculture the cells. The cell experiment protocols were performed in strict accordance with the Guidelines for Care and Use of Laboratory cells of China Japan Union Hospital of Jilin University and were approved by the Ethics Committee of China Japan Union Hospital of Jilin University.

\section{In vitro Cytotoxicity and Protection from Oxidative Stress Induced by $\mathrm{H}_{2} \mathrm{O}_{2}$}

The cytotoxicity of Se-CQDs was evaluated using an MTT assay against astrocytes, PC12, and N2a cells. ${ }^{27}$ Briefly, the cells (astrocytes, PC12 cells, or N2a) were seeded onto 96well plates at a density of 7000 cells/well in $200 \mu \mathrm{L}$ of DMEM, followed by incubation overnight. Next, the medium was completely aspirated, and $200 \mu \mathrm{L}$ of fresh media, containing different concentrations of Se-CQDs was added. After incubation for either 24 or $48 \mathrm{~h}$, MTT solution was added ( $20 \mu \mathrm{L} /$ well, $5 \mathrm{mg} / \mathrm{mL})$, and the cells were incubated for an additional $4 \mathrm{~h}$. Subsequently, the medium was removed and replaced with $150 \mu \mathrm{L}$ of dimethyl sulfoxide. The absorbance of each well was measured at $492 \mathrm{~nm}$, and cell viability ( $\%)$ was calculated as follows $\left(A_{\text {sample }} / A_{\text {control }}\right) \times$ $100 \%$, where $A_{\text {sample }}$ and $A_{\text {control }}$ are the absorbances of the sample and control wells, respectively.

To investigate the antioxidant ability of Se-CQDs, astrocytes, PC12, or N2a cells were seeded into 96-well plates at a density of 7000 cells per well. These were pre-treated with PBS or different concentrations 
(6.25-200 $\mu \mathrm{g} / \mathrm{mL})$ of Se-CQDs for $30 \mathrm{~min}$. Next, the cells were exposed to $250 \mu \mathrm{M} \mathrm{H}_{2} \mathrm{O}_{2}$ for $24 \mathrm{~h}$. Cell viability was determined using the MTT method. The viability of astrocytes, PC12, and N2a cells was also evaluated by the live/ dead cell staining method using commercial kits (04511; Sigma-Aldrich). Live/dead cells were observed by confocal laser scanning microscopy (CLSM) (Carl Zeiss LSM 780), and the live cell numbers were calculated using ImageJ software (NIH, Bethesda, MD, USA). ROS levels were quantitatively analyzed in astrocytes, $\mathrm{PC} 12$, and $\mathrm{N} 2 \mathrm{a}$ cells by analyzing the fluorescence intensity of $2^{\prime}, 7^{\prime}-$ dichlorofluorescein diacetate (DCFH-DA) (D6883, Sigma) using CLSM.

\section{Animal Model of TSCI}

Adult female Sprague-Dawley rats (200-250 g) were purchased from the Laboratory Animal Center of Jilin University. The rats were housed in controlledtemperature rooms $\left(23 \pm 2^{\circ} \mathrm{C}\right)$, under a $12 / 12 \mathrm{~h}$ lightdark cycle, with access to water and food ad libitum until use. ${ }^{28}$ The animal experiment protocols were performed in strict accordance with the Guidelines for Care and Use of Laboratory Animals of Jilin University and were approved by the Animal Ethics Committee of Jilin University.

The TSCI model was established using the weightdrop method. ${ }^{29}$ Briefly, rats were anesthetized by administering pentobarbital sodium, and a T10 laminectomy was conducted to expose the underlying thoracic spinal cord segment(s). A 40-g rod (2.5 $\mathrm{mm}$ in diameter) was dropped from a height of $40 \mathrm{~mm}$ to strike the exposed dorsal surface of the spinal cord. The muscle, fascia, and skin were closed in layers, followed by sterilization of the outer skin with iodophor. The criteria for successful modeling were as follows: The two lower extremities rapidly showed retraction-like shaking; the spinal dura mater at the impact site was intact, swollen, and hemorrhagic (Figure S1); and the two lower extremities showed complete delayed paralysis after waking up. ${ }^{30}$ Following surgery, the rats were returned to their cages and had free access to water and food. The rats were administered cefazolin $(25 \mathrm{mg} / \mathrm{kg})$ twice a day for 5 days to limit infection. ${ }^{31}$ The bladder was massaged twice daily until bladder function was restored.

\section{Behavioral Analysis}

The rats were randomly divided into four groups. The group without any surgery or treatment was set as the control, while the other three groups $(\mathrm{n}=9$ for each group) were separately treated (intraspinal injection) with saline, $2.5 \mu \mathrm{g}$ Se-CQDs, and $10 \mu \mathrm{g}$ Se-CQDs after establishment of the TSCI model. The Basso, Beattie, Bresnahan (BBB) locomotor rating score was used to evaluate the restoration of the hind limb locomotor function in an open and quiet container. ${ }^{32}$ At predetermined time points ( 1 day or 1-8 weeks post-injury), two independent researchers conducted the tests, and the scores were finalized when both researchers were in agreement. At the same time, the body weight and the time for the postoperative recovery of the urinary function of the rats were also recorded.

\section{Histological Analysis and Immunohistochemistry}

The rats were anesthetized and perfused with PBS and 4\% paraformaldehyde. A 2-cm segment of the spinal cord tissue was collected, fixed with $4 \%$ paraformaldehyde, embedded in paraffin, and sectioned in the coronal plane. Next, 4- $\mu$ m-thick tissue sections were used for hematoxylin and eosin (H\&E) staining. To evaluate the extent of myelination, $0.1 \%$ Luxol fast blue (LFB) stain was used for staining the sections. Then, the sections were examined under an optical microscope (Pannoramic DESK, P-MIDI, P250, P1000, 3D HISTECH, Budapest, Hungary). The relative LFB staining was evaluated with ImageJ software. To investigate the therapeutic effect of the different treatments on demyelination, the ultrastructure of the spinal cord was observed using transmission electron microscope (TEM) (Hitachi, HT7700). The dissected spinal cord segments were cut into $1-\mathrm{mm}^{3}$ slabs, fixed with $2.5 \%$ glutaraldehyde overnight at $4^{\circ} \mathrm{C}$, osmicated (90 min), and dehydrated (135 min). After embedding and sectioning, ultrathin sections were stained with uranyl acetate and lead citrate, and then used for TEM observations. The G-ratio (the ratio of inner axonal diameter to the outer axonal diameter, used to assess axonal myelination) was evaluated with ImageJ software.

To analyze the expression of NeuN, NF200, GFAP, CS56, and CD68, immunofluorescence staining assays were conducted using corresponding monoclonal primary antibody and fluorescently labeled secondary antibody or fluorescently labeled primary antibody. Antibody dilution information in the immunofluorescent staining is listed in Table S1, and then imaged by CLSM. Paraffin sections were permeabilized with PBS containing $0.1 \%$ Triton 
X-100 for 15 min, blocked with PBS containing 3\% bovine serum albumin for $1 \mathrm{~h}$, and incubated with primary antibodies overnight at $4{ }^{\circ} \mathrm{C}$. The primary antibodies antiNF200, anti-NeuN, anti-GFAP, and anti-CS56 were used to identify axons, neurons, astrocytes, and chondroitin sulfate, respectively. The sections were then incubated with secondary antibodies for $2 \mathrm{~h}$ at room temperature before staining with DAPI to label the nuclei. Then, the sections were washed and mounted before observation by CLSM.

\section{Anti-Apoptotic Effect of Se-CQDs on Secondary Injury in vivo}

We next evaluated the apoptosis-inhibitory effects of SeCQDs following secondary injury associated with TSCI. At $30 \mathrm{~min}$ post-contusion, saline and Se-CQDs $(1 \mathrm{mg} / \mathrm{mL}$, $10 \mu \mathrm{g}$ ) were injected into the lesion site at the T10 spinal cord. The rats were anesthetized and perfused with cold PBS at $24 \mathrm{~h}$ post-injection. T9-T10 spinal cord tissues (length, $1 \mathrm{~cm}$ ) containing the injury site were harvested and homogenized. The levels of cleaved caspase-3, caspase-9, Bcl-2, and Bax were evaluated by Western blotting. Western blotting was conducted with whole-spinal cord lysates, as previously described. ${ }^{33}$ Briefly, the spinal cord tissues were ground at a ratio of $0.1 \mathrm{~g}$ tissue $/ 0.1 \mathrm{~mL}$ $1 \mathrm{X}$ cell lysis buffer supplemented with proteinase inhibitors and centrifuged at 13,300 rpm for $10 \mathrm{~min}$; then, the supernatants were collected. To quantify the protein lysates, a BCA protein kit (Beyotime, Shanghai, China) was used. Equal amounts of proteins $(10 \mu \mathrm{g})$ were electrophoresed on $10 \%$ sodium dodecyl sulfate polyacrylamide gels and then transferred onto polyvinylidene fluoride membranes (Millipore). The membranes were blocked using 5\% bovine serum albumin for $1.5 \mathrm{~h}$. Next, they were incubated with primary antibodies at $4^{\circ} \mathrm{C}$ overnight, and then with secondary antibodies at room temperature for $2 \mathrm{~h}$. GAPDH was used as an internal reference. Antibody dilution information in the Western blotting is listed in Table S2. The images of the protein bands were scanned using an enhanced chemiluminescence Western blot detection system (GE AI600). The intensities of the bands on the blots were quantified using Multi Gauge software (Fuji, Tokyo, Japan).

\section{Statistical Analysis}

All experiments were performed a minimum of three times, and the data are shown as the mean \pm standard deviations.
The statistical significance of results was analyzed by oneway analysis of variance or $t$ tests in GraphPad Prism Software (GraphPad, 8.0.2, Inc., San Diego, CA, USA). $\mathrm{P}<0.05$ was considered statistically significant.

\section{Results and Discussion Preparation and Characterization of Se-CQDs}

The water-soluble Se-CQDs were facilely prepared via the hydrothermal treatment of L-selenocystine according to a previously described method. ${ }^{19}$ The hydrodynamic radius $\left(R_{\mathrm{h}}\right)$ of the Se-CQDs was $34.5 \pm 3.3 \mathrm{~nm}$ (Figure 2A). Morphological analysis by TEM demonstrated that the Se-CQDs had a spherical shape, with an average diameter of approximately $40 \mathrm{~nm}$ (Figure 2B). The relatively smaller particle sizes compared with those measured by DLS might be attributed to the shrinkage of the shell during the drying procedure in the TEM sample preparation. ${ }^{19,26}$ The zeta potential of the Se-CQDs was $-14.2 \pm 0.47$. The molecular structure of the Se-CQDs was further characterized by NMR and FT IR spectroscopy.
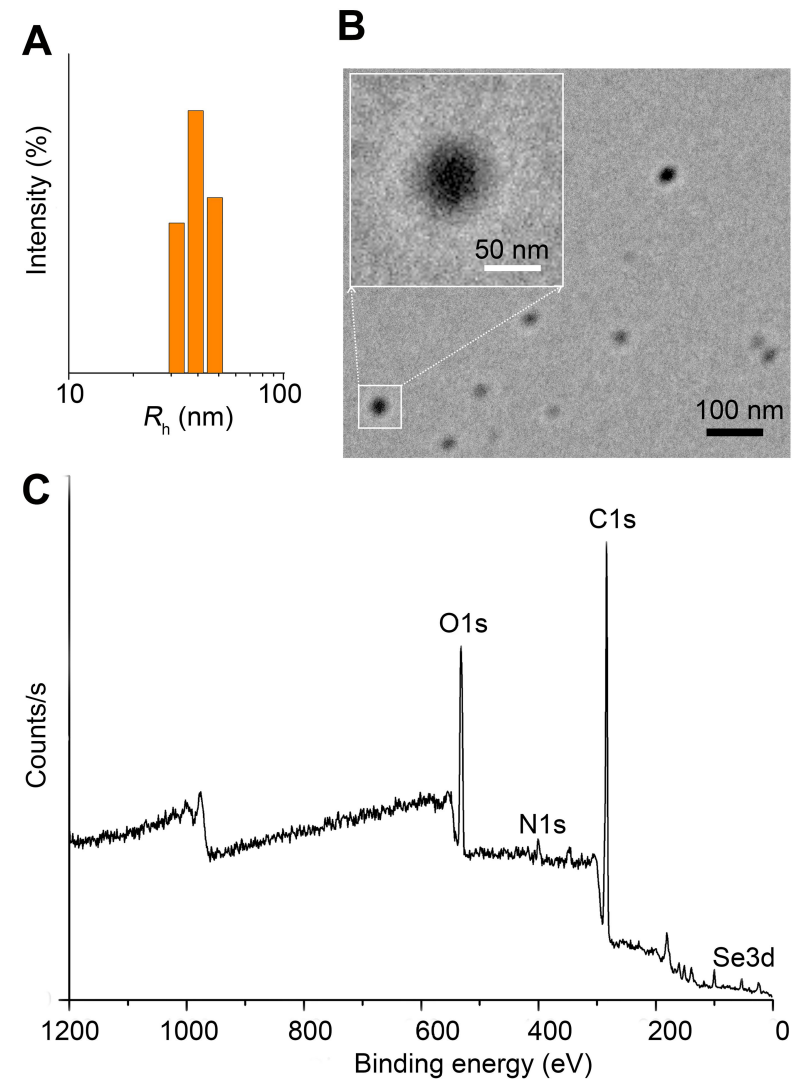

Figure 2 Characterization of Se-CQDs. (A) Particle size of the Se-CQDs, as measured by DLS. (B) TEM image of Se-CQDs. (C) XPS spectrum of Se-CQDs. 
The ${ }^{1} \mathrm{H}$ NMR, ${ }^{13} \mathrm{C}$ NMR, and FTIR spectra (Figures S2, $\underline{\mathrm{S} 3}$ and S4) indicated that the Se-CQDs had different chemical structures from those of the precursor molecule, L-selenocystine, which confirmed the chemical transformation of L-selenocystine into Se-CQDs. The XPS spectrum indicated that the Se-CQDs were mainly composed of carbon, nitrogen, selenium, and oxygen, which corresponded to the typical peaks of $\mathrm{C} 1 \mathrm{~s}, \mathrm{~N} 1 \mathrm{~s}, \mathrm{O} 1 \mathrm{~s}$, and $\mathrm{Se}$ 3d (Figure 2C). The Se-CQDs displayed a broad absorption in the UV/Vis absorption spectra (Figure S5) and a strong emission at $480 \mathrm{~nm}$ (Figure S6), which were consistent with the previous reports. ${ }^{34-37}$ All the structural characterizations are consistent with the previous report. ${ }^{19}$

\section{Biocompatibility and Antioxidation Effect of Se-CQDs}

Previous reports have suggested that Se-CQDs or other carbon dots were low toxic and biocompatible. ${ }^{34,38}$ To assess the potential application of the synthesized Se-CQDs in biological systems, cytotoxicity tests were first performed to investigate their biocompatibility. The cell viabilities of astrocytes, $\mathrm{PC} 12$, and N2a cells were assessed at 24 or $48 \mathrm{~h}$ after exposure to $0-200 \mathrm{mg} / \mathrm{L}$ of Se-CQDs by the MTT assay. The results showed that the Se-CQDs exhibited no observable cytotoxicity under $200 \mathrm{mg} / \mathrm{L}$ of Se-CQDs (Figure S7).

It is well documented that ROS overproduction plays a vital role in the secondary injury cascade after acute TSCI. $^{20,23}$ To simulate the ROS-induced oxidative damage to cells, $\mathrm{H}_{2} \mathrm{O}_{2}$ was added into the cell culture medium, ${ }^{24}$ and apparent cell death was observed after $24 \mathrm{~h}$ of culture (Figure $3 \mathrm{~A}$ and Figure S8A). Then, the antioxidation effect of SeCQDs was investigated in astrocytes and N2a cells upon treatment with $250 \mu \mathrm{M} \mathrm{H}_{2} \mathrm{O}_{2}$. As shown in Figure 3B and Figure S8B, the cell viability of astrocytes and N2a cells increased with the increase in Se-CQDs, indicating that SeCQDs could significantly reduce $\mathrm{H}_{2} \mathrm{O}_{2}$-induced cell death, which is consistent with the previous report. ${ }^{39}$ Moreover, the intracellular ROS levels with or without treatment with SeCQDs were investigated, and the results are shown in Figure 3C and 3D. After treatment with Se-CQDs, the fluorescence intensity of DCF was significantly reduced, indicating the effective reduction of ROS levels by Se-CQDs. A similar result could be seen when using PC12 and N2a cells as the tested cells (Figures S9A, S9B, and Figure S8C). Furthermore, the protective capacity of the Se-CQDs against ROS-induced oxidative damage was further confirmed by live/dead cell staining (Figure 3E, 3F, S8D, S9C, and S9D).
The live/dead cell staining results also demonstrated that there were more viable cells in groups treated with SeCQDs, consistent with the results of the MTT assay (Figure 3B). Taken together, these results suggest that the Se-CQDs can effectively scavenge ROS and protect cells from oxidative stress-induced damage.

\section{Therapeutic Effect of Se-CQDs in a Rat Model of TSCl}

In view of the outstanding anti-oxidation effect of SeCQDs in vitro, we further investigated the therapeutic effect of Se-CQDs in a rat model of contusion TSCI. The Se-CQDs were administrated via intraspinal injection. The BBB score test was used to evaluate the behavioral function of rats with TSCI. At 8 weeks post-operation, the BBB scores of rats from the $2.5 \mu \mathrm{g}$ Se-CQDs treated group $(9.80 \pm 0.68)$ and $10 \mu \mathrm{g}$ Se-CQDs treated group (12.40 \pm $0.45)$ were significantly higher than those of the rats from the saline-treated group $\left(\begin{array}{lll}6.80 & \pm 0.37\end{array}\right)$ (Figure 4A). Moreover, the rats treated with $10 \mu \mathrm{g}$ Se-CQDs showed a better therapeutic outcome compared to those treated with $2.5 \mu \mathrm{g}$ Se-CQDs. Specifically, rats from the salinetreated group showed a sweeping hind limb without weight support, while rats in the Se-CQDs treated groups were able to take frequent weight-supported plantar steps and showed occasional coordination of the hind limb with the forelimb (Figure 4B). In addition, the lesion area in the spinal cord from the Se-CQDs treated rats was smaller than that from the saline-treated group (Figure 4C). Collectively, these results demonstrated that Se-CQDs could reduce the secondary injury and promote the recovery of locomotor function in TSCI rats.

Injury of the spinal cord leads to the development of neurogenic bladder, a fatal complication in patients with TSCI. ${ }^{24,40}$ In this study, the reduction in secondary injury by Se-CQDs treatment was expected to be beneficial for the protection of bladder tissues. As shown in Figure 4D, the rats in the Se-CQDs treated groups regained spontaneous urination faster than those in the saline-treated group. To provide more evidence for the recovery of the urination function, H\&E and Masson staining of the bladder tissues were performed. Pathological damage to the bladder tissue was found to be remarkably restrained after treatment with Se-CQDs, with reduced levels of bladder endometriosis, cell vacuolation, and bladder wall fibrosis compared with the bladder tissues from rats in the salinetreated group (Figure 4E and 4F). In addition, no 
A

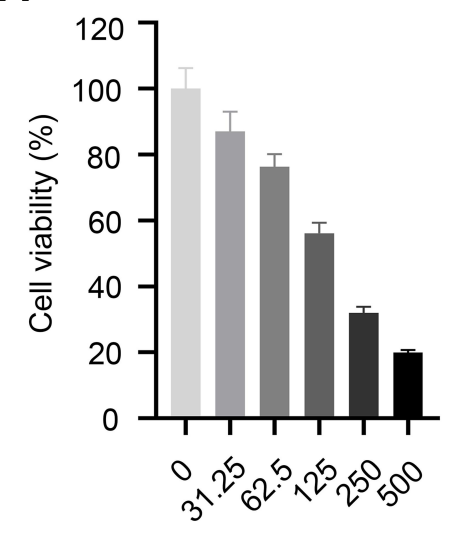

Concentration $(\mu \mathrm{M})$
B

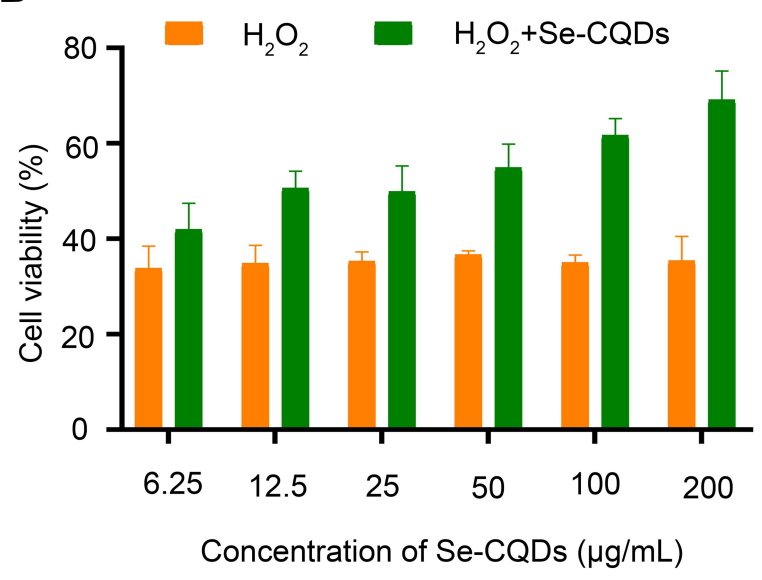

D

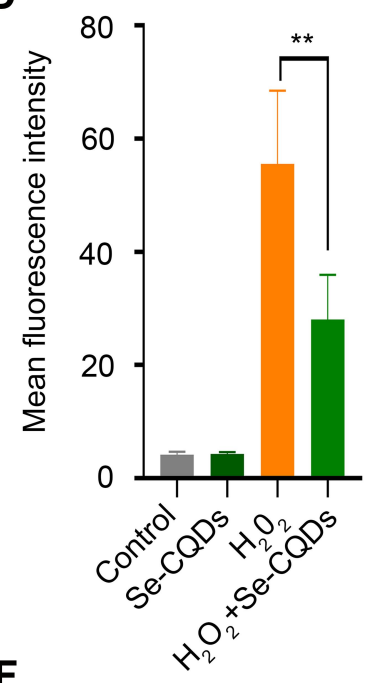

$\mathbf{F}$
C

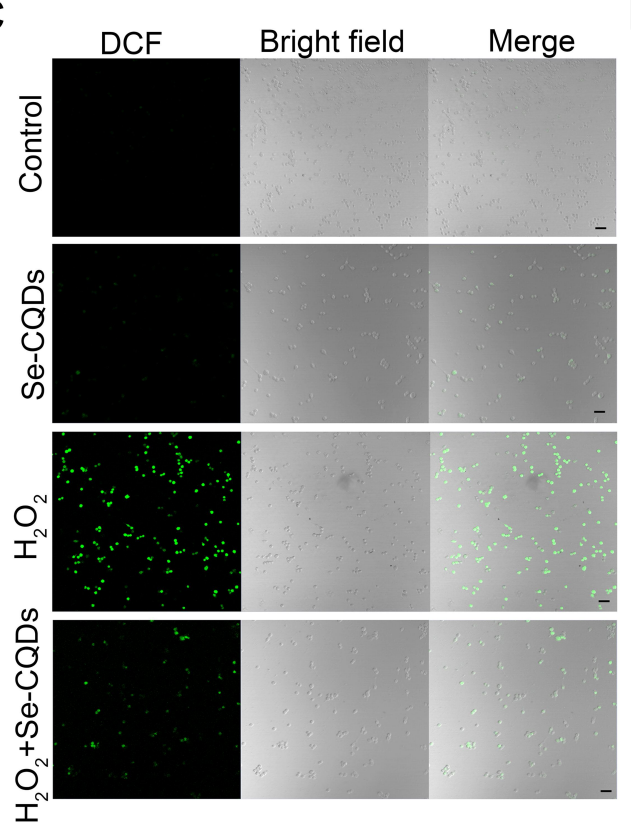

E

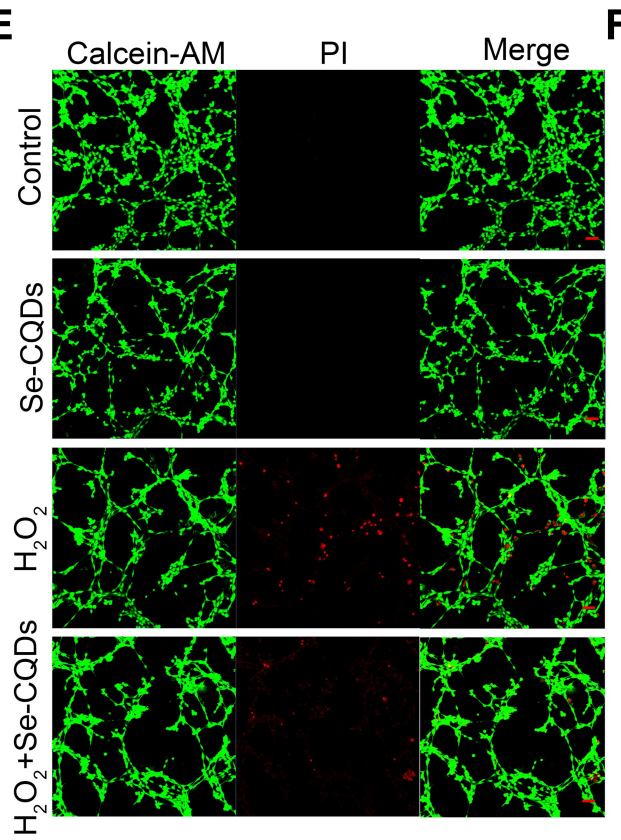

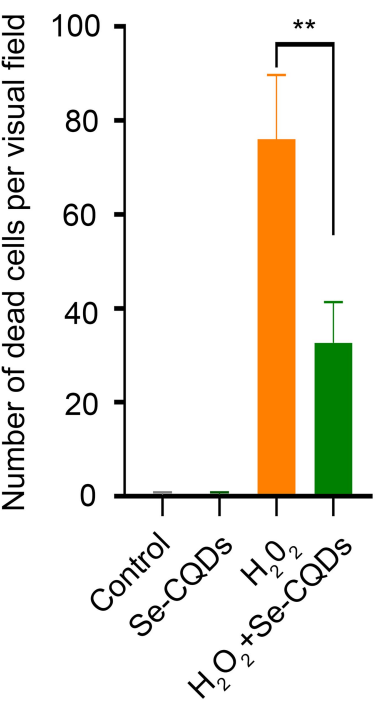

Figure $3 \mathrm{Se}-\mathrm{CQDs}$ scavenge ROS to protect astrocytes from ROS-induced oxidative damage. (A) Effect of $\mathrm{H}_{2} \mathrm{O}_{2}$ on the viability of astrocytes. (B) Protective effect of SeCQDs against $\mathrm{H}_{2} \mathrm{O}_{2}$-induced oxidative damage in astrocytes. The concentration of $\mathrm{H}_{2} \mathrm{O}_{2}$ was $250 \mu \mathrm{M}$. (C) Intracellular ROS levels in astrocytes were measured by DCF staining. (D) Quantitative analysis of the fluorescence intensity of DCF in Figure $2 \mathrm{C}, * * \mathrm{P}<0.0 \mathrm{I}$, when + Se-CQDs group compared with $\mathrm{H}_{2} \mathrm{O}_{2}$ group. (E) Live/dead straining of astrocytes under different conditions, scale bar $=20 \mu \mathrm{m}$. (F) Quantitative analysis of the number of dead cells in Figure $2 \mathrm{E}$, $* * \mathrm{P}<0.0 \mathrm{I}$, when $+\mathrm{Se}-\mathrm{CQDs}$ group compared with $\mathrm{H}_{2} \mathrm{O}_{2}$ group.

differences in body weight were observed between the rats from the saline, $2.5 \mu \mathrm{g}$ Se-CQDs, and $10 \mu \mathrm{g}$ Se-CQDs treated groups (Figure S10), which indicated that the SeCQDs showed no obvious adverse effects.

\section{Histomorphological Changes in Spinal Cords in Different Treatment Groups}

To provide more evidence on the recovery of locomotor function, H\&E staining was performed to study the histomorphological changes in the spinal cord at 8 weeks postinjury. The integrity and continuity of the spinal cord in the rats from the saline-treated group were the poorest, and severe lesion cavities were observed in this group (Figure $5 \mathrm{~A}$ ). Following treatment with different concentrations of Se-CQDs, the integrity of the spinal cord was wellpreserved, and inflammatory cell infiltration was mild. TSCI frequently causes severe axonal demyelination and myelin structural impairment. ${ }^{4,51-43}$ We next evaluated the level of axonal demyelination and the ultrastructure of myelin sheaths at the lesion sites by LFB staining and TEM, respectively (Figure 5B and 5C). The ratio of inner axonal diameter to the outer axonal diameter, denoted as the 
A

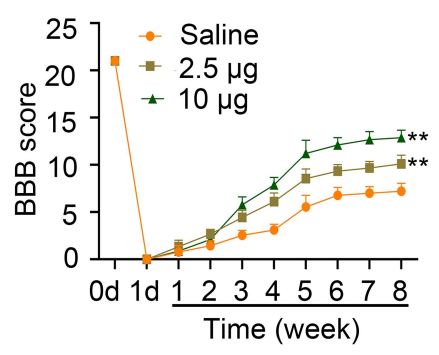

D

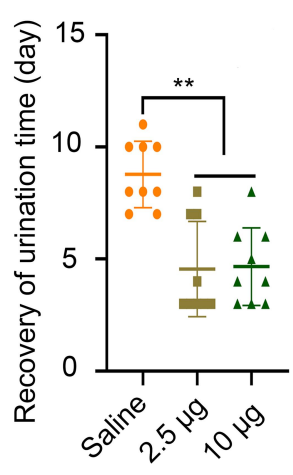

E
B
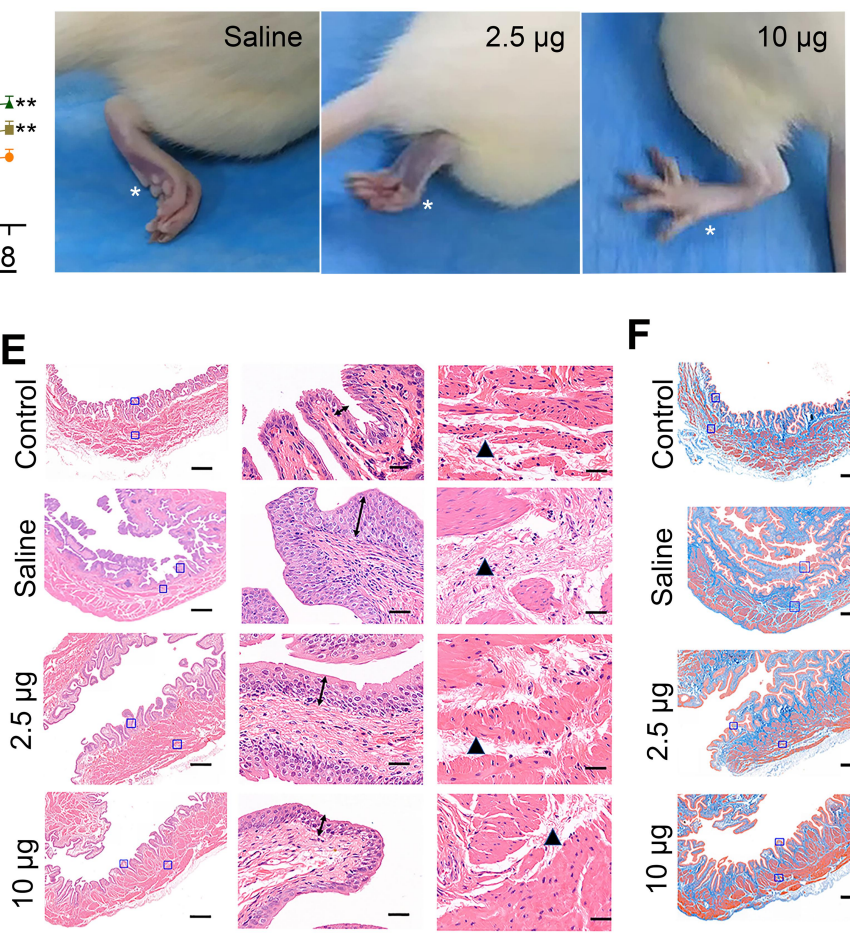

C

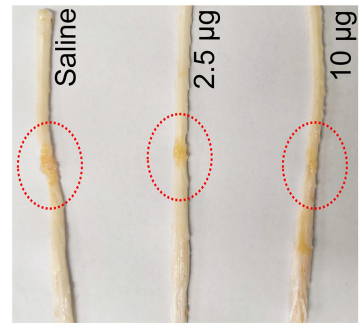

F

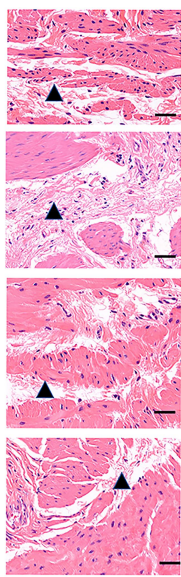

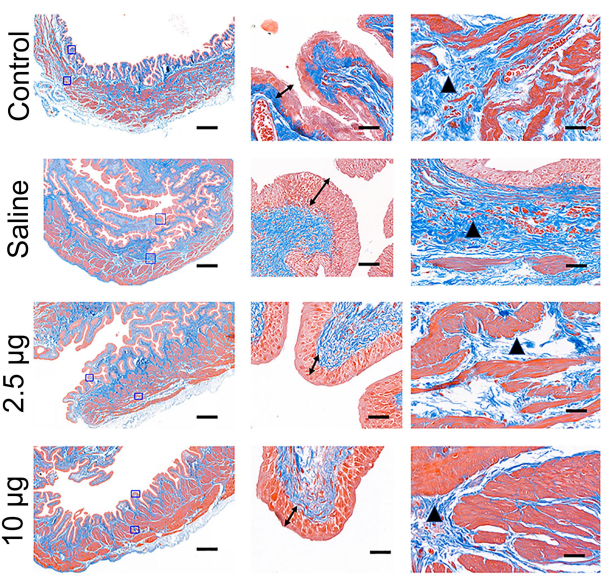

Figure 4 Assessment of locomotor functional recovery following traumatic spinal cord injury (TSCI). (A) BBB scores of rats with TSCl treated with saline, $2.5 \mu \mathrm{g}$ Se-CQDs, or $10 \mu \mathrm{g}$ Se-CQDs. ** $\mathrm{P}<0.0 \mathrm{I}$, in comparison with the saline group. (B) Typical images of the foot in rats with TSCl after treatment with saline, $2.5 \mu \mathrm{g}$ Se-CQDs, or $10 \mu \mathrm{g}$ Se-CQDs. The asterisk * indicates the hindlimb walking patterns. (C) Typical images of spinal cords collected from rats in the saline, $2.5 \mu \mathrm{g}$ Se-CQDs, or I0 $\mu$ g Se-CQDs treated groups at 8 weeks post-injury. The red circles indicate the lesion sites. (D) Recovery of the urination time of rats with TSCl after treatment with saline, $2.5 \mu \mathrm{g}$ SeCQDs, or $10 \mu \mathrm{g}$ Se-CQDs ** $\mathrm{P}<0.0 \mathrm{I}$, when + Se-CQDs group was compared with saline group. (E) H\&E staining of bladders shown at $2 \times$ (left) and $20 \times$ magnification (right), and the followed two images show the magnified views of the fields marked by the blue squares, arrows and triangles indicated the pathological characteristics. (F) Masson staining of bladders shown at $2 \times$ (left) and $20 \times$ magnification (right). In the Figure 4E and 4F, the two images on the right are the magnified views of the fields marked by the blue squares in the leftmost image; arrows and triangles indicated the hyperplasia and fibrosis characteristics of bladder, respectively. The scale bars are $500 \mu \mathrm{m}$ and 50 $\mu \mathrm{m}$ for the images obtained at $2 \times$ and $20 \times$ magnification, respectively.

G-ratio, was used to assess the axonal myelination. ${ }^{41}$ The results of relative LFB staining and G-ratio are shown in Figure S11. The samples from rats in the saline treatment group displayed notable demyelination (Figure 5B and Figure S11 A) and impaired myelin sheath ultrastructure (Figure S11 B) compared with those from rats in the control group, suggesting that significant demyelination occurred at 8 weeks post-injury. In contrast, more myelin sheaths with better ultrastructure were preserved in the Se-CQDs-treated group, and the higher dose of administered Se-CQDs resulted in the better preservation of myelin sheaths. Taken together, these results revealed that Se-CQDs can reduce the demyelination of nerve fibers in the lesion site. Furthermore, the neurons and axons in the injured spinal cord were analyzed by staining the tissue slices with anti-NeuN and anti-NF200 antibodies, respectively. As shown in Figure 5D, $5 \mathrm{E}$ and $5 \mathrm{~F}$ the rats treated with saline showed marked neuronal loss at the lesion sites after TSCI. In contrast, the rats from the Se-CQDs treatment groups showed higher numbers of neurons, suggesting that Se-CQDs therapy could improve the neuronal survival.

\section{Anti-Inflammatory Effect of Se-CQDs and Their Ability to Reduce Glial Scars and Apoptosis in vivo}

It is suggested that ROS scavenging can suppress the inflammation in secondary injury following TSCI. ${ }^{21,44}$ Therefore, we investigated the in vivo anti-inflammatory properties of the Se-CQDs at 5 days post operation. The immunostaining of the spinal cord showed an increase in the numbers of cells positive for CD68 (a marker of microglia) in the lesion sites of rats from the saline treatment group. In contrast, fewer CD68-positive cells were observed in the samples from the Se-CQDs treatment groups (Figure 6A), indicating that SeCQDs could effectively reduce the inflammation in the injured site. Meanwhile, the overproduction of ROS results 
A

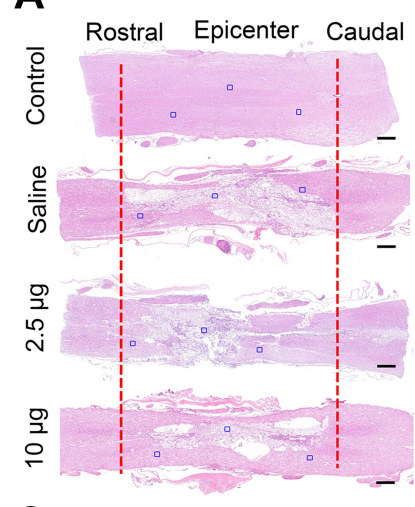

C

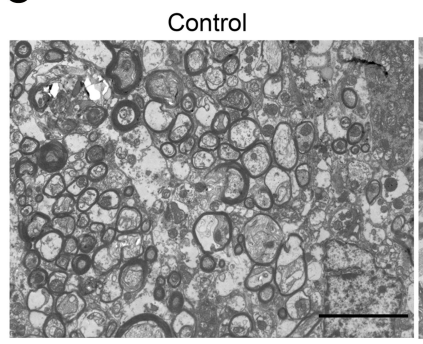

D

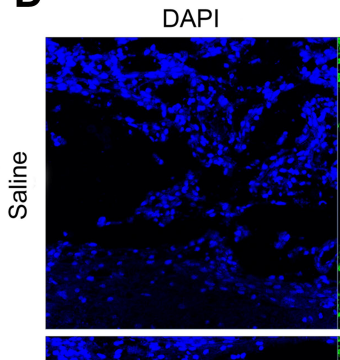

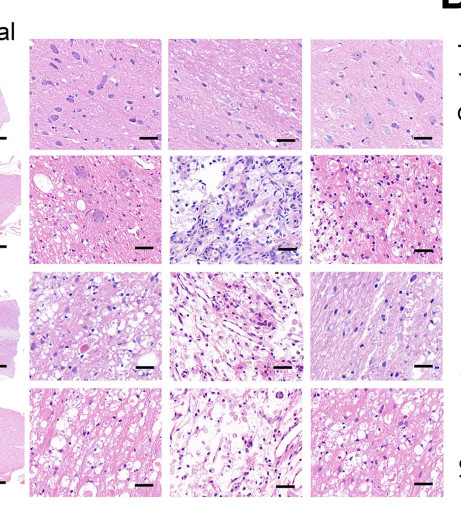

B

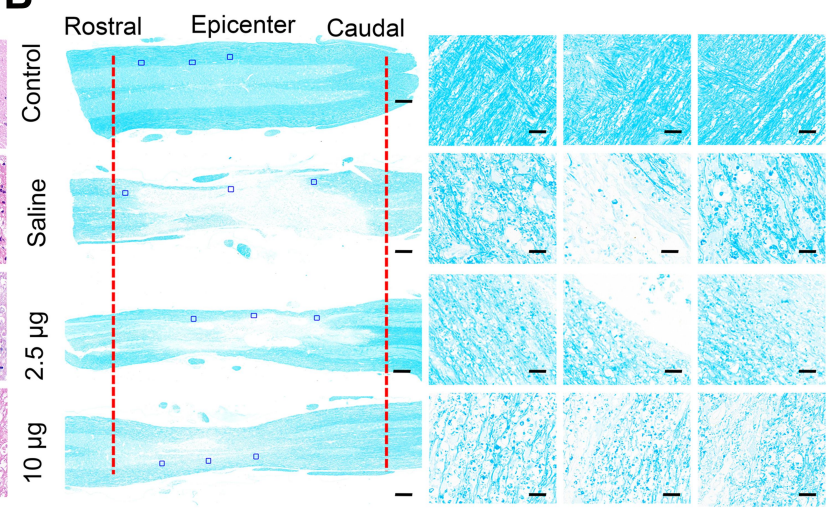

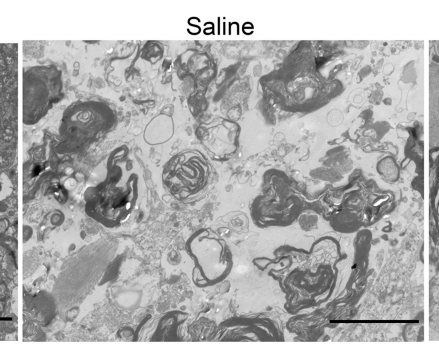

NF200

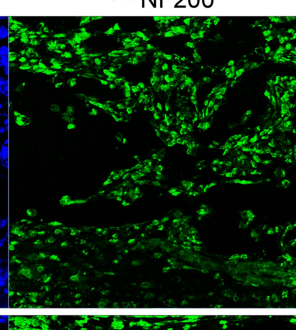

NeuN

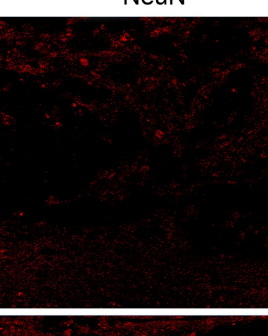

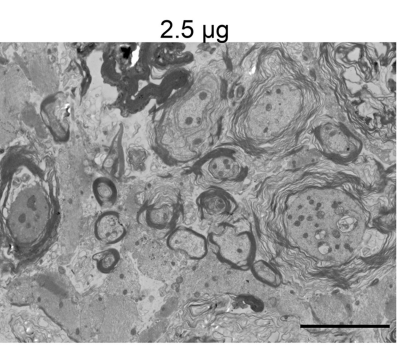

Merge
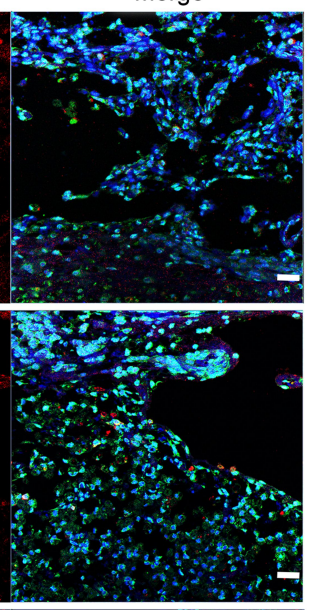

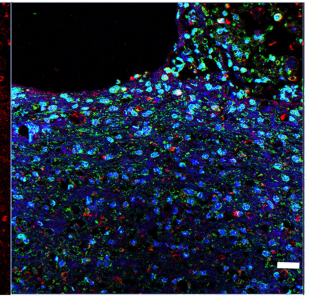

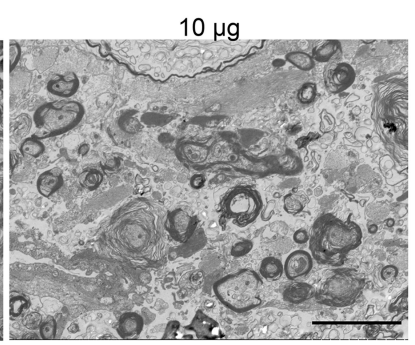

E

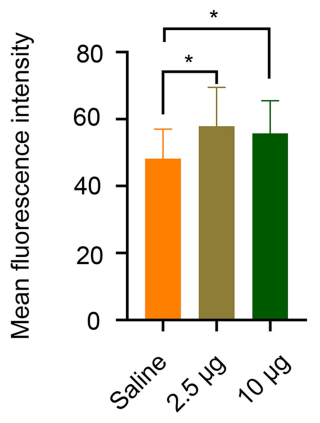

F

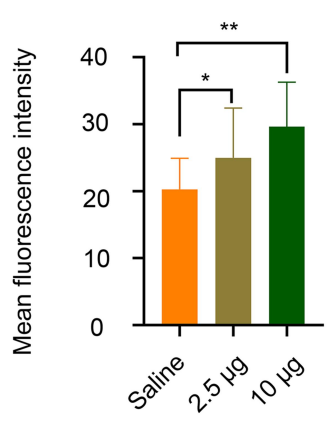

Figure 5 Histomorphological changes at 8 weeks post-injury in vivo. (A) Images of spinal cord tissue after staining with $\mathrm{H} \& \mathrm{E}$, shown at $2 \mathrm{X}$ (left, scale bar $=500 \mu \mathrm{m}$ ) and 20 $X$ magnification (right, scale bar $=50 \mu \mathrm{m}$ ). The images on the right are the corresponding enlarged views of the areas indicated by blue squares in the left images. The red lines indicate the edge of the lesion sites. (B) LFB staining of myelin sheaths is shown at $2 X$ (left, scale bar $=500 \mu \mathrm{m})$ and $20 \mathrm{X}$ magnification (right, scale bar $=50 \mu \mathrm{m})$. The images on the right are the corresponding enlarged views of the areas indicated by blue squares in the left images. The red lines indicate the edge of the lesion sites. (C) Representative TEM images showing the ultrastructure of myelin sheaths in different groups. Scale bar $=5 \mu \mathrm{m}$. (D) Double-staining of the slices with anti-NF200 and antiNeuN antibodies. Scale bar $=50 \mu \mathrm{m}$. (E) Quantitative analysis of mean fluorescence intensity of NF200 staining in Figure 4D, * P< 0.05 , in comparison with saline group. (F) Quantitative analysis of mean fluorescence intensity of NeuN staining in Figure 4D. $* P<0.05$ and ** $P<0.0$, in comparison with saline group. 
in glutamate-mediated excitotoxicity, which activates astrocytes via their glutamate receptors. ${ }^{45}$ Then, the activated astrocytes secrete chondroitin sulfate (CS56), which results in the formation of a dense glia scar. ${ }^{46-49}$ The formation of glial scars can interrupt neural pathways of the spinal cord and suppress the recovery of locomotor function. ${ }^{39,49-52}$ Therefore, we evaluated the formation of glial scars by the co-immunostaining of CS56 and GFAP (indicating the activated astrocytes ${ }^{53,54}$ ) at 8 weeks after the operation. As compared with the saline-treated group, the Se-CQDstreated groups showed a significant reduction in CS56 and GFAP expression (Figure 6B), indicating that the scavenging of ROS by Se-CQDs could effectively reduce the activation of astrocytes and the formation of glial scars. ${ }^{53,54}$
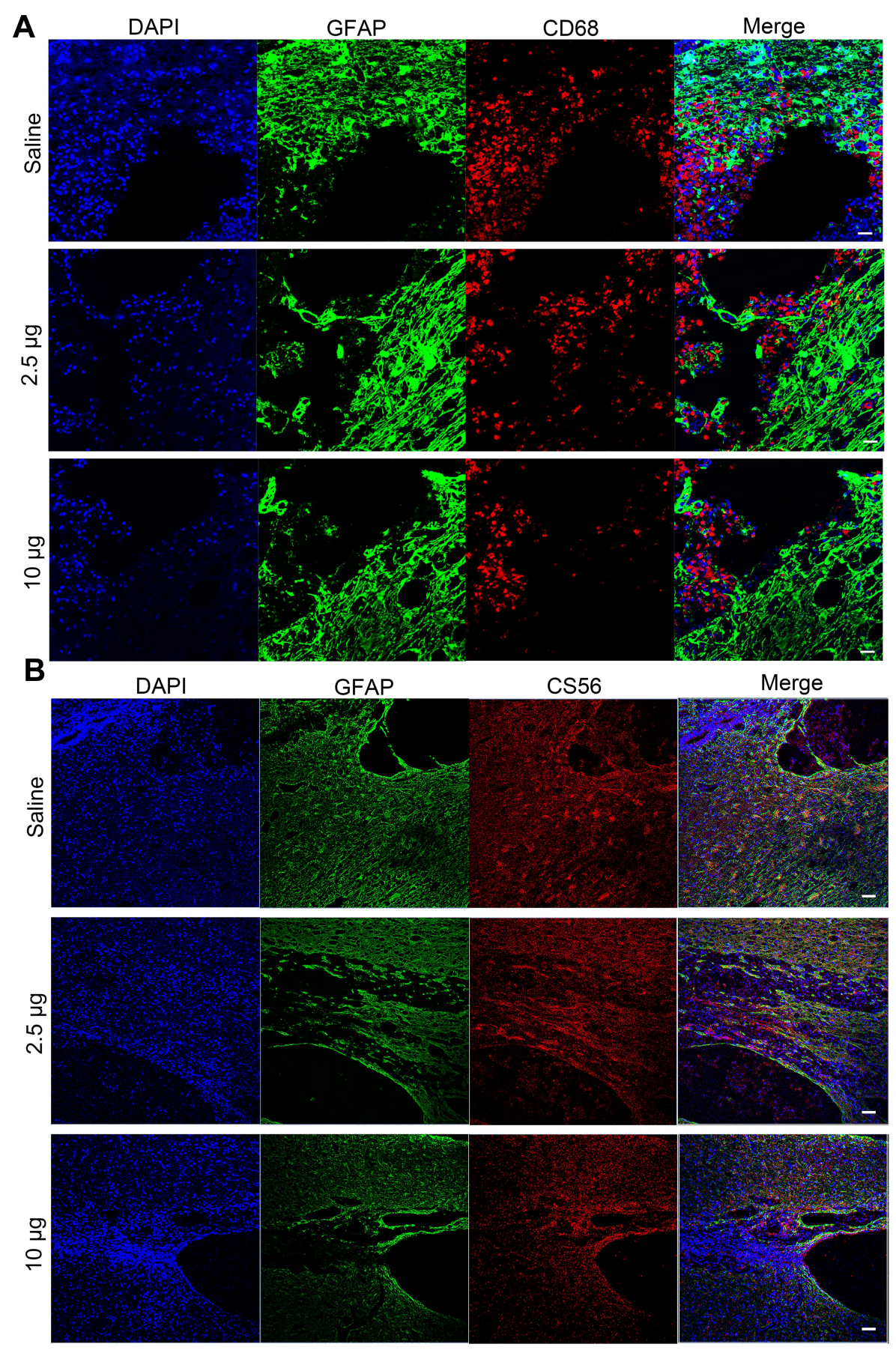

Figure 6 Immunofluorescence images showing the mechanism underlying the Se-CQDs-mediated promotion of behavioral functional recovery. (A) Immunofluorescence images highlighting the anti-inflammatory properties of Se-CQDs. Scale bar $=50 \mu \mathrm{m}$. (B) Immunofluorescence images showing the inhibition of glial scars. Scale bar $=50 \mu \mathrm{m}$. 

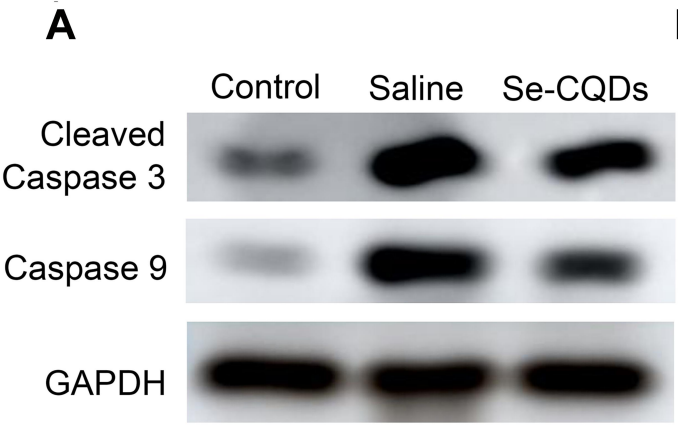

D

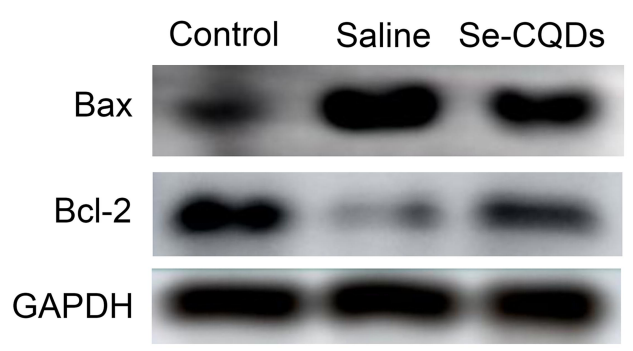

B

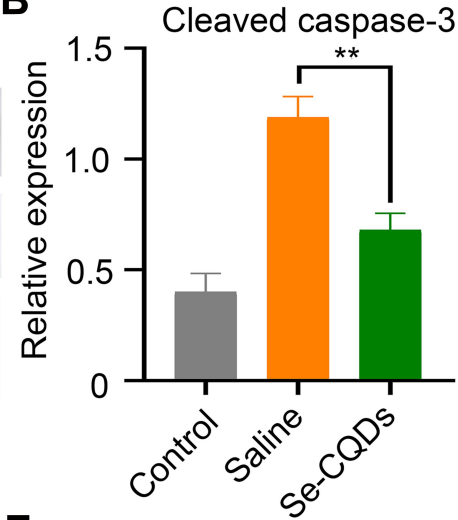

E

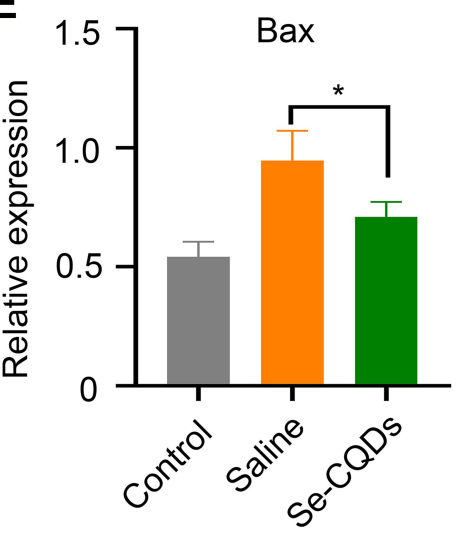

C

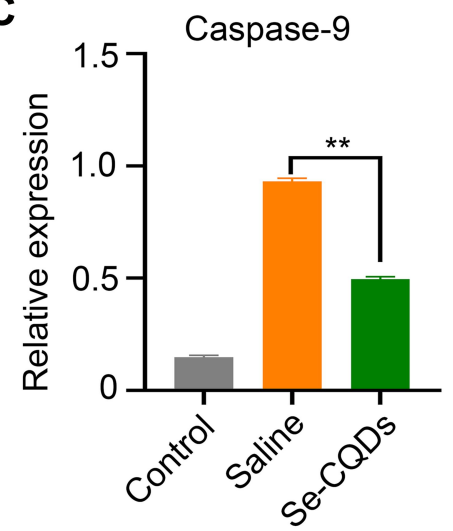

F

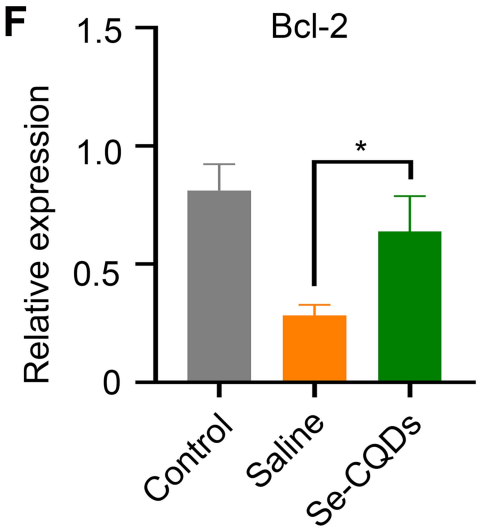

Figure 7 Anti-apoptotic effects of Se-CQDs. (A) Western blot analysis of cleaved caspase-3 and caspase-9 protein levels in the injured spinal cord tissues from different groups. (B) Semi-quantitative analysis of cleaved caspase-3 protein levels based on the data in Figure 6A, ** P<0.0I, in comparison with the saline group. (C) Semiquantitative analysis of caspase- 9 protein levels based on the data in Figure $6 \mathrm{~A}$, ** $\mathrm{P}<0.01$, in comparison with the saline group. (D) Western blot analysis of Bax and Bcl-2 protein levels. (E) Semi-quantitative analysis of Bax protein levels based on the data in Figure 6D, * $P<0.05$, in comparison with the saline group. (F) Semi-quantitative analysis of $\mathrm{Bcl}-2$ protein levels based on the data in Figure $6 \mathrm{D}, * \mathrm{P}<0.05$, in comparison with the saline group. Data are expressed as the mean $\pm \mathrm{SD}$; $\mathrm{n}=3$.

The long-term neurological deficits following spinal cord trauma partially result from the widespread activation of the apoptotic pathway in neurons and oligodendroglia at TSCI lesion sites. ${ }^{43}$ To evaluate the ability of Se-CQDs to inhibit apoptosis following TSCI, we measured the protein expression of cleaved caspase-3, caspase-9, Bax, and Bcl-2 at the lesion sites (Figure 7). At $24 \mathrm{~h}$ post injury, cleaved caspase3, Bax, and caspase-9 expression levels were significantly decreased in the rats receiving Se-CQDs treatment, compared to those in the saline-treated group. In contrast, Bcl-2 expression was increased. All these results suggested that Se-CQDs exerted remarkable anti-apoptotic effects following TSCI, which might be beneficial for locomotor recovery of TSCI rats after treatment with Se-CQDs.

\section{Conclusions}

In this study, a type of water-soluble Se-CQDs was prepared in a quick and easy manner through the hydrothermal treatment of L-selenocystine. The obtained Se-CQDs displayed good biocompatibility and a remarkable protective effect against
$\mathrm{H}_{2} \mathrm{O}_{2}$-induced oxidative damage to astrocytes, PC12, and N2a cells. The protection effect should be ascribed to the excellent ROS-scavenging ability of Se-CQDs. ${ }^{39,55}$ As a result, these Se-CQDs reduced the inflammation, astrogliosis, and apoptosis induced by secondary injury, which ultimately led to effective restoration of locomotor function in TSCI rats. In general, our findings suggest that Se-CQDs may serve as a promising therapeutic strategy for TSCI treatment by attenuating the exacerbation of the secondary injury cascade after TSCI. Moreover, due to the robust ROS-scavenging ability, Se-CQDs may be promising for use in the treatment of other diseases characterized with up-regulated ROS. However, it should be noted that the biosafety and detailed mechanism need to be carefully investigated before clinical use of SeCQDs.

\section{Acknowledgments}

We acknowledge the financial support from the National Natural Science Foundation of China (51803072, 51773196, and 51573184), Jilin Provincial Science and 
Technology Program (20180520207JH), Jilin Provincial Finance Program (2019SCZ023, 2018SCZ013), and the Bethune Plan Research Project of Jilin University (2018B15 and 470110000648). We are very grateful to Prof. Jiaoqi Wang for providing the PC12 cells.

\section{Disclosure}

The authors report no conflicts of interest for this work.

\section{References}

1. David G, Mohammadi S, Martin AR, et al. Traumatic and nontraumatic spinal cord injury: pathological insights from neuroimaging. Nat Rev Neurol. 2019;15(12):718-731. doi:10.1038/s41582-0190270-5

2. Ge L, Arul K, Ikpeze T, et al. Traumatic and Nontraumatic Spinal Cord Injuries. World Neurosurg. 2018;111:e142-e8. doi:10.1016/j. wneu.2017.12.008

3. Hara M, Kobayakawa $\mathrm{K}$, Ohkawa $\mathrm{Y}$, et al. Interaction of reactive astrocytes with type I collagen induces astrocytic scar formation through the integrin-N-cadherin pathway after spinal cord injury. Nat Med. 2017;23(7):818-828. doi:10.1038/nm.4354

4. Song YH, Agrawal NK, Griffin JM, et al. Recent advances in nanotherapeutic strategies for spinal cord injury repair. Adv Drug Deliv Rev. 2019;148:38-59. doi:10.1016/j.addr.2018.12.011

5. Ahuja CS, Wilson JR, Nori S, et al. Traumatic spinal cord injury. Nat Rev Dis Primers. 2017;3:17018.

6. Fischer I, Dulin JN, Lane MA. Transplanting neural progenitor cells to restore connectivity after spinal cord injury. Nat Rev Neurosci. 2020;21:366-383.

7. Singh A, Tetreault L, Kalsi-Ryan S, et al. Global prevalence and incidence of traumatic spinal cord injury. Clin Epidemiol. 2014; 6:309-331.

8. Rubiano AM, Carney N, Chesnut R, et al. Global neurotrauma research challenges and opportunities. Nature. 2015;527:S193.

9. Shi Y, Kim S, Huff TB, et al. Effective repair of traumatically injured spinal cord by nanoscale block copolymer micelles. Nat Nanotechnol. 2009;5:80-87.

10. Tran AP, Warren PM, Silver J. The Biology of Regeneration Failure and Success After Spinal Cord Injury. Physiol Rev. 2018;98 (2):881-917. doi:10.1152/physrev.00017.2017

11. Tyler JY, Xu XM, Cheng JX. Nanomedicine for treating spinal cord injury. Nanoscale. 2013;5(19):8821-8836. doi:10.1039/c3nr00957b

12. He Z, Zang H, Zhu L, et al. An anti-inflammatory peptide and brain-derived neurotrophic factor-modified hyaluronan-methylcellulose hydrogel promotes nerve regeneration in rats with spinal cord injury. Int J Nanomedicine. 2019;14:721-732. doi:10.2147/IJN.S187854

13. Zhou X, Shi G, Fan B, et al. Polycaprolactone electrospun fiber scaffold loaded with iPSCs-NSCs and ASCs as a novel tissue engineering scaffold for the treatment of spinal cord injury. Int J Nanomedicine. 2018;13:6265-6277. doi:10.2147/IJN.S175914

14. Huang H, Young W, Skaper S, et al. Clinical Neurorestorative Therapeutic Guidelines for Spinal Cord Injury (IANR/CANR version 2019). J Orthop Translat. 2020;20:14-24. doi:10.1016/j.jot.2019. 10.006

15. Walters BC, Hadley MN, Hurlbert RJ, et al. Guidelines for the management of acute cervical spine and spinal cord injuries: 2013 update. Neurosurgery. 2013;60(CN_suppl_1):82-91. doi:10.1227/01. neu.0000430319.32247.7f

16. Silva NA, Sousa N, Reis RL, et al. From basics to clinical: a comprehensive review on spinal cord injury. Prog Neurobiol. 2014;114:25-57. doi:10.1016/j.pneurobio.2013.11.002
17. Visavadiya NP, Patel SP, VanRooyen JL, et al. Cellular and subcellular oxidative stress parameters following severe spinal cord injury. Redox Biol. 2016;8:59-67. doi:10.1016/j.redox.2015.12.011

18. Zhong G, Yang X, Jiang X, et al. Dopamine-melanin nanoparticles scavenge reactive oxygen and nitrogen species and activate autophagy for osteoarthritis therapy. Nanoscale. 2019;11(24):1160 5-11616. doi:10.1039/C9NR03060C

19. Li F, Li T, Sun C, et al. Selenium-Doped Carbon Quantum Dots for Free-Radical Scavenging. Angew Chem Int Ed Engl. 2017;56 (33):9910-9914. doi:10.1002/anie.201705989

20. Nukolova NV, Aleksashkin AD, Abakumova TO, et al. Multilayer polyion complex nanoformulations of superoxide dismutase 1 for acute spinal cord injury. J Control Release. 2018;270:226-236. doi:10.1016/j.jconrel.2017.11.044

21. Kim JW, Mahapatra C, Hong JY, et al. Functional Recovery of Contused Spinal Cord in Rat with the Injection of Optimal-Dosed Cerium Oxide Nanoparticles. Adv Sci. 2017;4:1700034. doi:10.1002/ advs.201700034

22. Baranes K, Shevach M, Shefi O, et al. Gold Nanoparticle-Decorated Scaffolds Promote Neuronal Differentiation and Maturation. Nano Lett. 2016;16(5):2916-2920. doi:10.1021/acs.nanolett.5b04033

23. Andrabi SS, Yang J, Gao Y, et al. Nanoparticles with antioxidant enzymes protect injured spinal cord from neuronal cell apoptosis by attenuating mitochondrial dysfunction. $J$ Control Release. 2020;317:300-311. doi:10.1016/j.jconrel.2019.12.001

24. Li L, Xiao B, Mu J, et al. A MnO2 Nanoparticle-Dotted Hydrogel Promotes Spinal Cord Repair via Regulating Reactive Oxygen Species Microenvironment and Synergizing with Mesenchymal Stem Cells. ACS Nano. 2019;13:14283-14293. doi:10.1021/acsnano. 9b07598

25. Zhang $\mathrm{Y}$, $\mathrm{Li} \mathrm{L}, \mathrm{Mu} \mathrm{J}$, et al. Implantation of a functional TEMPO-hydrogel induces recovery from rat spinal cord transection through promoting nerve regeneration and protecting bladder tissue. Biomater Sci. 2020;8(6):1695-1701. doi:10.1039/C9BM01530B

26. Huang G, Lin Y, Zhang L, et al. Synthesis of Sulfur-Selenium Doped Carbon Quantum Dots for Biological Imaging and Scavenging Reactive Oxygen Species. Sci Rep. 2019;9(1):19651. doi:10.1038/ s41598-019-55996-w

27. Shen W, Liu W, Yang $\mathrm{H}$, et al. A glutathione-responsive sulfur dioxide polymer prodrug as a nanocarrier for combating drug-resistance in cancer chemotherapy. Biomaterials. 2018; 178:706-719. doi:10.1016/j.biomaterials.2018.02.011

28. Choi B, Soh M, Manandhar Y, et al. Highly selective microglial uptake of ceria-zirconia nanoparticles for enhanced analgesic treatment of neuropathic pain. Nanoscale. 2019;11(41):19437-19447. doi:10.1039/C9NR02648G

29. Wang XJ, Shu GF, Xu XL, et al. Combinational protective therapy for spinal cord injury medicated by sialic acid-driven and polyethylene glycol based micelles. Biomaterials. 2019;217:119326. doi:10.1016/j.biomaterials.2019.119326

30. Young W. Spinal cord contusion models. Prog Brain Res. 2002; 137:231-255.

31. Guo S, Perets N, Betzer O, et al. Intranasal Delivery of Mesenchymal Stem Cell Derived Exosomes Loaded with Phosphatase and Tensin Homolog siRNA Repairs Complete Spinal Cord Injury. ACS Nano. 2019;13(9):10015-10028. doi:10.1021/acsnano.9b01892

32. Basso DM, Beattie MS, Bresnahan JC. A sensitive and reliable locomotor rating scale for open field testing in rats. $J$ Neurotrauma. 1995;12(1):1-21. doi:10.1089/neu.1995.12.1

33. Wang H, Wang R, Cai K, et al. Selective in vivo metabolic cell-labeling-mediated cancer targeting. Nat Chem Biol. 2017;13 (4):415-424. doi:10.1038/nchembio.2297

34. Ashrafizadeh M, Mohammadinejad R, Kailasa SK, et al. Carbon dots as versatile nanoarchitectures for the treatment of neurological disorders and their theranostic applications: A review. Adv Colloid Interface Sci. 2020;278:102123. doi:10.1016/j.cis.2020.102123 
35. Mewada A, Pandey S, Shinde S, et al. Green synthesis of biocompatible carbon dots using aqueous extract of Trapa bispinosa peel. Mater Sci Eng C Mater Biol Appl. 2013;33(5):2914-2917. doi:10. 1016/j.msec.2013.03.018

36. Mehta VN, Jha S, Kailasa SK. One-pot green synthesis of carbon dots by using Saccharum officinarum juice for fluorescent imaging of bacteria (Escherichia coli) and yeast (Saccharomyces cerevisiae) cells. Mater Sci Eng C Mater Biol Appl. 2014;38:20-27. doi:10. 1016/j.msec.2014.01.038

37. D'Souza SL, Deshmukh B, Bhamore JR, et al. Synthesis of fluorescent nitrogen-doped carbon dots from dried shrimps for cell imaging and boldine drug delivery system. RSC Adv. 2016;6(15): 12169-12179. doi:10.1039/C5RA24621K

38. Mehta VN, Jha S, Basu H, et al. One-step hydrothermal approach to fabricate carbon dots from apple juice for imaging of mycobacterium and fungal cells. Sens Actuators B Chem. 2015;213:434-443. doi:10.1016/j.snb.2015.02.104

39. Rosenkrans ZT, Sun T, Jiang D, et al. Selenium-Doped Carbon Quantum Dots Act as Broad-Spectrum Antioxidants for Acute Kidney Injury Management. Adv Sci. 2020;7:2000420. doi:10.1002/ advs.202000420

40. Hamid R, Averbeck MA, Chiang H, et al. Epidemiology and pathophysiology of neurogenic bladder after spinal cord injury. World J Urol. 2018;36(10):1517-1527. doi:10.1007/s00345-018-2301-z

41. Wang XJ, Peng CH, Zhang S, et al. Polysialic-Acid-Based Micelles Promote Neural Regeneration in Spinal Cord Injury Therapy. Nano Lett. 2019;19(2):829-838. doi:10.1021/acs.nanolett.8b04020

42. Saab AS, Nave KA. Neuroscience: A mechanism for myelin injury. Nature. 2016;529(7587):474-475. doi:10.1038/nature16865

43. Zhang S, Wang XJ, Li WS, et al. Polycaprolactone/polysialic acid hybrid, multifunctional nanofiber scaffolds for treatment of spinal cord injury. Acta Biomater. 2018;77:15-27. doi:10.1016/j.actbio.20 18.06.038

44. Gao Y, Vijayaraghavalu S, Stees M, et al. Evaluating accessibility of intravenously administered nanoparticles at the lesion site in rat and pig contusion models of spinal cord injury. $J$ Control Release. 2019;302:160-168. doi:10.1016/j.jconrel.2019.03.026
45. Peng JJ, Lin SH, Liu YT, et al. A circuit-dependent ROS feedback loop mediates glutamate excitotoxicity to sculpt the Drosophila motor system. Elife. 2019;8:e47372.

46. Fuhrmann T, Anandakumaran PN, Shoichet MS. Combinatorial Therapies After Spinal Cord Injury: how Can Biomaterials Help? Adv Healthc Mater. 2017;6.

47. Sofroniew MV. Dissecting spinal cord regeneration. Nature. 2018;557:343-350.

48. Brochier C, Jones JI, Willis DE, et al. Poly(ADP-ribose) polymerase 1 is a novel target to promote axonal regeneration. Proc Natl Acad Sci U S A. 2015;112:15220-15225.

49. Zou Y, Ma D, Shen H, et al. Aligned collagen scaffold combination with human spinal cord-derived neural stem cells to improve spinal cord injury repair. Biomater Sci. 2020;8(18):5145-5156.

50. Moeendarbary E, Weber IP, Sheridan GK, et al. The soft mechanical signature of glial scars in the central nervous system. Nat Commun. 2017;8:14787.

51. Vismara I, Papa S, Veneruso V, et al. Selective Modulation of A1 Astrocytes by Drug-Loaded Nano-Structured Gel in Spinal Cord Injury. ACS Nano. 2020;14:360-371.

52. Mayo L, Trauger SA, Blain M, et al. Regulation of astrocyte activation by glycolipids drives chronic CNS inflammation. Nat Med. 2014;20:1147-1156.

53. Anderson MA, Burda JE, Ren Y, et al. Astrocyte scar formation aids central nervous system axon regeneration. Nature. 2016;5 32:195-200.

54. Kamermans A, Planting KE, Jalink K, et al. Reactive astrocytes in multiple sclerosis impair neuronal outgrowth through TRPM7-mediated chondroitin sulfate proteoglycan production. Glia. 2019;67:68-77.

55. Yao H, Tang X, Shao X, et al. Parthenolide protects human lens epithelial cells from oxidative stress-induced apoptosis via inhibition of activation of caspase-3 and caspase-9. Cell Res. 2007;17:565-571.
International Journal of Nanomedicine

\section{Publish your work in this journal}

The International Journal of Nanomedicine is an international, peerreviewed journal focusing on the application of nanotechnology in diagnostics, therapeutics, and drug delivery systems throughout the biomedical field. This journal is indexed on PubMed Central, MedLine, CAS, SciSearch ${ }^{\circledR}$, Current Contents ${ }^{\circledR} /$ Clinical Medicine,
Journal Citation Reports/Science Edition, EMBase, Scopus and the Elsevier Bibliographic databases. The manuscript management system is completely online and includes a very quick and fair peer-review system, which is all easy to use. Visit http://www.dovepress.com/ testimonials.php to read real quotes from published authors. 